\title{
Pneumatosis Intestinalis Presenting as Small Bowel Obstruction without Bowel Ischemia after Mechanical Ventilation
}

\author{
Dong Joon Kim', Yong Joon Choi', Young Sun Yoo ${ }^{2}$ \\ Departments of ${ }^{1}$ Anesthesiology and Pain Medicine and ${ }^{2}$ Surgery, Chosun University Hospital, Gwangju, Korea
}

Pneumatosis intestinalis $(\mathrm{PI})$ is a rare condition of the presence of gas within the bowel walls. $\mathrm{PI}$ is associated with numerous underlying diseases, ranging from life-threatening to innocuous conditions. $\mathrm{Pl}$ is believed to be secondary to coexisting disorders in approximately $85 \%$ of all cases. This paper reviews the case of a patient who was diagnosed 7 years prior with pneumoperitoneum from unknown causes without any symptoms. The patient was admitted to the intensive care unit for the management of aspiration pneumonia and developed extensive Pl after mechanical ventilation, presenting as small bowel obstruction with mesenteric torsion. Although the exact mechanism and etiology of $\mathrm{PI}$ are unclear, this case provides an update on the imaging features of and the clinical conditions associated with $\mathrm{Pl}$, as well as the management of this condition.

Key Words: intestinal obstruction; pneumatosis intestinalis; pulmonary emphysema

\section{Case Report}

Received: April 18, 2016

Revised: September 12, 2016

Accepted: September 28, 2016

Corresponding author

Young Sun Yoo

Department of Surgery, Chosun

University Hospital, 365 Pilmun-

daero, Dong-gu, Gwangju 61453,

Korea

Tel: +82-62-220-3676

Fax: +82-62-228-3441

E-mail:ysyoo@chosun.ac.kr

Pneumatosis intestinalis (PI) is described as the presence of gas confined within the bowel walls. PI is rare but has recently increased clinical attention due to the frequent use of computed tomography (CT) scans and regular health screening. Others have mentioned the radiographic incidence of PI to be $0.37 \%$ in patients who undergo abdominal CT scans [1]. New medications and surgical procedures are reportedly associated with an increased PI incidence. PI is associated with numerous underlying diseases. Primary PI represents $15 \%$ of cases, while secondary PI represents $85 \%$ of cases [2]. Because the initial symptoms of PI are not prominent, the condition tends to be severe when diagnosed, and emergent surgery is necessary for life-threatening PI. However, various conservative treatment measures may be employed if the condition is diagnosed early.

PI can be secondary to pulmonary disorders; however, there are few reports of association with mechanical ventilation. We present the case of a 71-year-old man admitted to the intensive care unit (ICU) for the treatment of aspiration pneumonia, who developed extensive PI after mechanical ventilation, presenting as small bowel obstruction with mesenteric torsion. This report presents a rare case of massive PI causing small bowel obstruction with intraperitoneal free air without bowel ischemia probably secondary to mechanical ventilation in a patient who was already diagnosed with pulmonary emphysema and asymptomatic pneumoperitoneum 7 years prior.
Copyright @ 2019 The Korean Society of Critical Care Medicine

This is an Open Access article distributed under the terms of Creative Attributions Non-Commercial License (http:// creativecommons.org/li-censes/by-nc/4.0/ which permits unrestricted noncommercial use, distribution, and reproduction in any medium, provided the original work is properly cited. 


\section{CASE REPORT}

A 71-year-old man (height, $169 \mathrm{~cm}$; weight, $68 \mathrm{~kg}$ ) with a medical history of lung emphysema was admitted to the ICU for treatment of aspiration pneumonia. He was already diagnosed with pneumoperitoneum from PI 7 years prior (Figure 1), but was asymptomatic and did not show obvious surgical indications. Mechanical ventilation was performed for 4 days to treat respiratory distress and hypoxemia. The ventilator settings were set in pressure control ventilation, with an inspiratory pressure between 10 to $20 \mathrm{cmH}_{2} \mathrm{O}$, modified by the ideal body weight of $6 \mathrm{ml} / \mathrm{kg}$. The respiratory rate was 12 breaths/ min in the controlled ventilation mode. And the respiratory rate increased gradually to 20 breaths/min by changing the
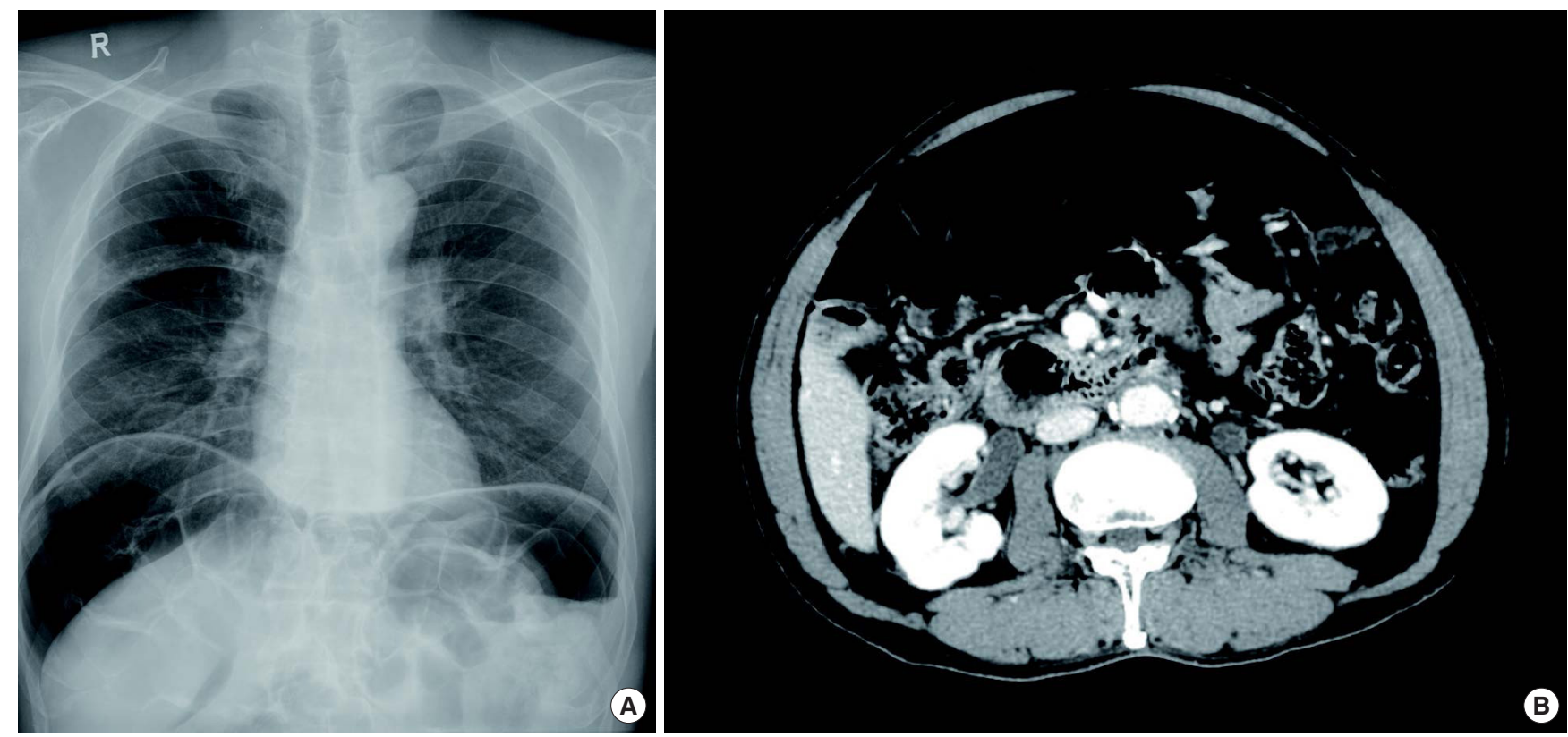

Figure 1. (A) Massive free air at both subdiaphragmatic areas. Mildly emphysematous lung on the right. (B) Large amount of free air in the abdominal cavity on computed tomography. Both figures had been taken 7 years previously.
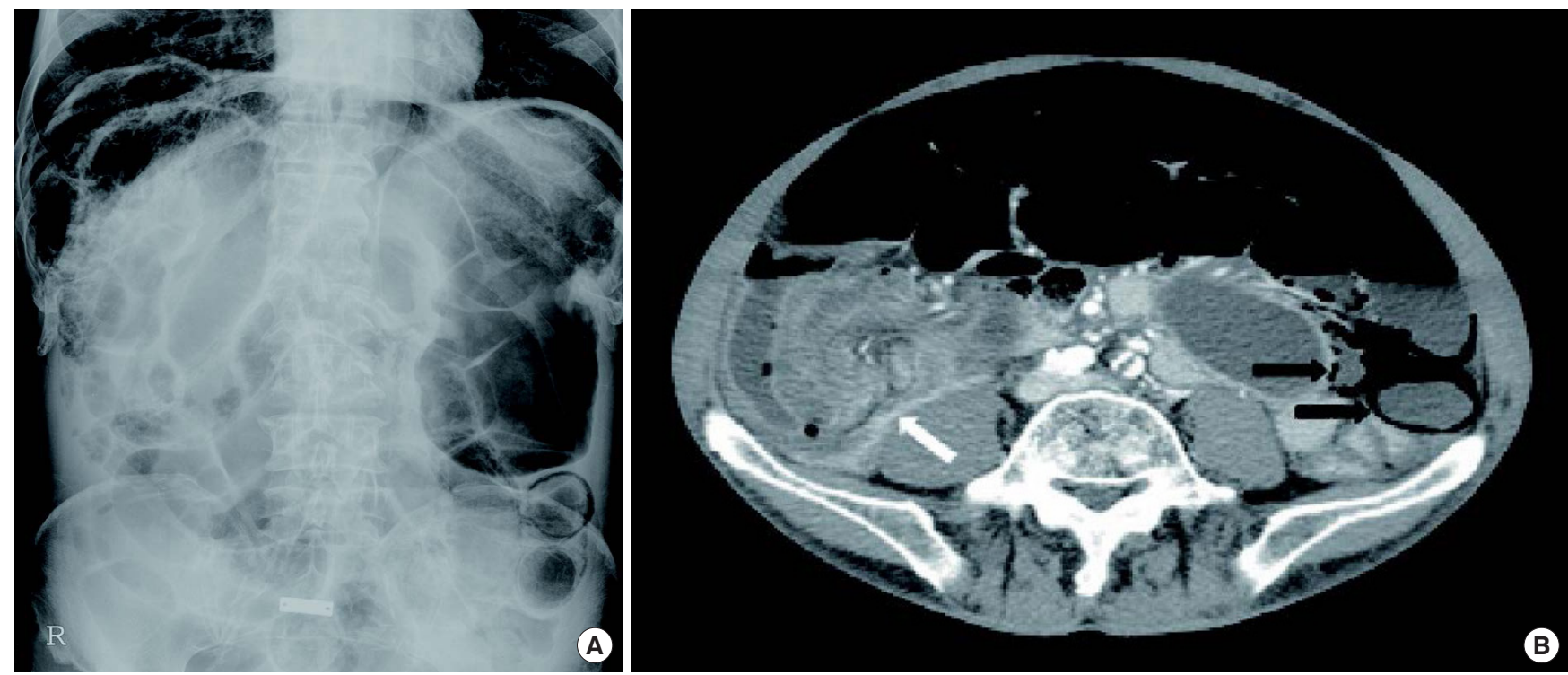

Figure 2. (A) Septated pneumoperitoneum at the right subphrenic area. Dilatation and pneumatosis intestinalis in the small bowel. (B) Segmental edematous small bowel wall thickening with air bubbles (black arrows), and mesenteric torsion showing a whirling sign (white arrow). 
ventilation mode to spontaneous ventilation mode. The positive end-expiratory pressure was minimized to $4 \mathrm{cmH}_{2} \mathrm{O}$ as prophylaxis, to avoid rupture of emphysematous lung bullae. On the day of mechanical ventilator weaning and extubation, the patient complained of sudden-onset pain and distension in the abdomen. On physical examination, the entire abdomen was rigid, strongly suspicious for acute abdomen. The plain abdominal radiograph (Figure 2A) and CT scan (Figure 2B) showed segmental edematous small bowel wall thickening with air-bubble and mesenteric torsion showing the whirling sign, and a moderate amount of ascites. While preparing for the emergency operation, his vital signs were as follows: blood pressure, 130/90 mmHg; pulse rate, 110 beats/min; respiratory rate, 26 breaths $/ \mathrm{min}$; and body temperature, $36.2^{\circ} \mathrm{C}$. The arterial blood gas analysis revealed the following results: $\mathrm{pH}$ 7.432; arterial partial pressure of carbon dioxide $\left(\mathrm{PaCO}_{2}\right)$ $34.8 \mathrm{mmHg}$; arterial partial pressure of oxygen $\left(\mathrm{PaO}_{2}\right) 58.6$
mmHg; and $\mathrm{O}_{2}$ saturation of $91.6 \%$ on mask oxygenation at 3 L/min. Laboratory diagnostics revealed the following: leuko-

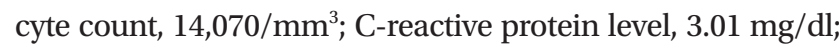
and lactate level, $2.5 \mathrm{mmol} / \mathrm{L}$. Other blood laboratory values were within reference ranges except aspartate aminotransferase (83.2 U/L) and alanine transaminase ( $42.4 \mathrm{U} / \mathrm{L})$. Emergency operation for mesenteric torsion was performed. No bowel ischemia or hollow viscus perforation was identified during the operation. Twisted small bowel loops and a stacked small bowel segment with large air bubbles were prominent in the right subphrenic space. The twisted and stacked small bowel loops were reduced and divided (Figure 3A, B).

After reduction, extensive and bulky PI extending from the proximal ileum to the terminal ileum was identified (Figure 3C). Although there was no obvious perforation, a total of 100 $\mathrm{cm}$ of small bowel with impending perforation was resected (Figure 3D). Sections from this specimen revealed numerous
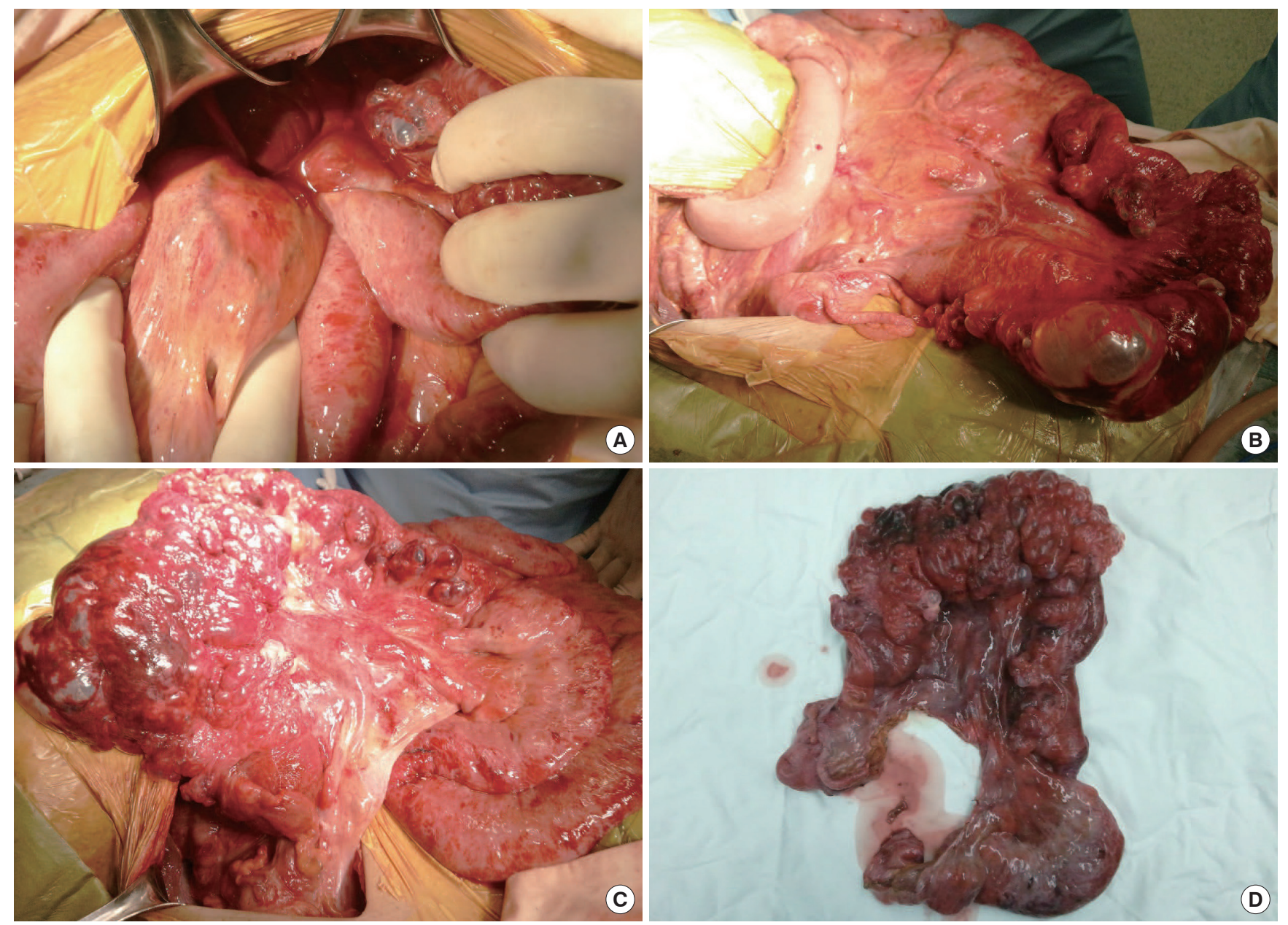

Figure 3. (A) Twisted small bowel loops in the right lower quadrant. (B) Reduction of stacked small bowel showing massive pneumatosis intestinalis. (C) The other side of the small bowel mesentery showing large bubbles on the small bowel wall. (D) Resected specimen showing extensive pneumatosis intestinalis. 


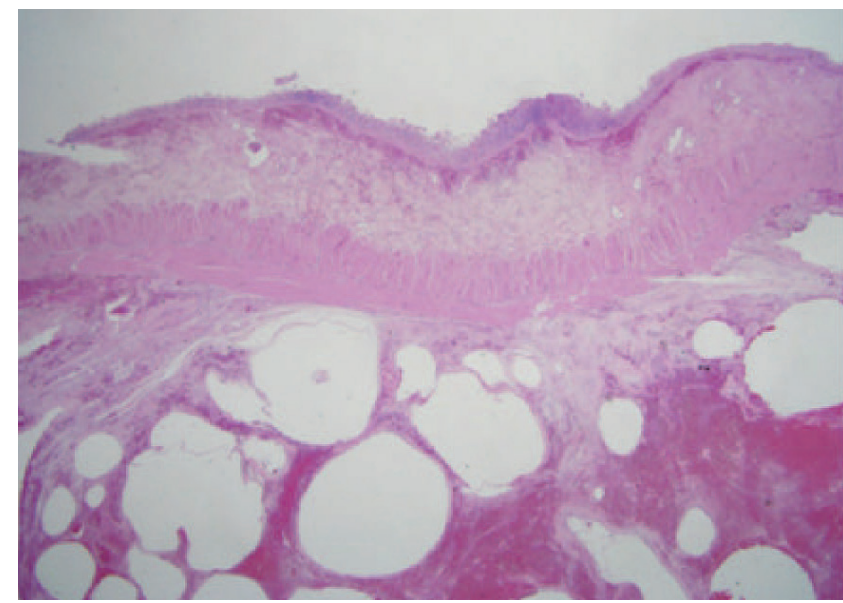

Figure 4. H\&E staining of small bowel $(\times 5)$. Numerous air pockets were observed in the subserosal layer.

subserosal air-pockets in small bowel wall (Figure 4). The postoperative course was uneventful, and the patient was discharged 2 weeks after surgery.

\section{DISCUSSION}

PI was first described in the literature by Du Vernoi in 1730, as the presence of extraluminal bowel gas that is confined within the bowel walls. Our patient was diagnosed with PI based on the radiographic and pathologic findings of air within the bowel wall. PI is rare; based on autopsy cases, the overall incidence has been reported to be $0.03 \%$. PI, has recently come to increased clinical attention due to increased CT scans and regular health screening.

The pathogenesis of PI is strongly debated; many theories exist. PI can be classified into two groups: primary PI (15\%), occurring without any apparent cause, and secondary PI (85\%), occurring in association with other diseases. Three possibilities are suggested as the source of the gas within the gastrointestinal tract walls: bacterial, mechanical, and pulmonary causes [3]. Primary PI is mostly asymptomatic; it involves the large bowel and heals without any complication. Secondary PI is associated with several etiological factors. Known associations include mechanical causes such as volvulus, ileus, Crohn disease, HIV, scleroderma, respiratory etiologies such as chronic obstructive pulmonary disease, drug toxicities, graft versus host disease, transplantation, and gut ischemia [4]. The small bowel (42\%) is the most common site for the development of PI, followed by the colon (36\%), with the involvement of both in $22 \%$ [5]. Although most are asymptomatic and discovered by chance. Improvements are observed even if treated con- servatively, but, in some cases, surgical treatment is required [4]. PI is mostly asymptomatic, but some cases present with diarrhea, hematochezia, abdominal pain, abdominal distension, weight loss, and tenesmus. In cases where large bubbles of bowel occur, ileus, malrotation, volvulus, and intussusception can be present [6].

Intraperitoneal free air can be seen even in the absence of bowel perforation; this makes it difficult for the surgeon to decide whether an emergency operation is necessary. Proper management directed towards a host of clinical diseases with a wide range of severity is necessary, and some algorithm indicates the management methods [7]. It is, however, very important to decide whether an emergency operation is indicated, because PI that requires emergency surgery shows a relatively high mortality rate. Greenstein et al. [7] suggested that the indications for emergency operation in patients with evidence of mesenteric torsion or bowel obstruction, after careful evaluation of the patients' clinical state and diagnostic results, are a white blood cell count $>12,000 / \mathrm{mm}^{3}$ and portal vein gas on CT, especially those ages $>60$ years.

In patients admitted to the hospital because of abdominal pain and diagnosed with PI on CT scan, the decision on whether a quick operation is necessary or not is important, and affects the prognosis, physiological outcome and clinical course of the patient. Thus, physicians and surgeons must be aware of these factors and their effects on the prognosis, and must carefully examine the patient. Although PI can be managed conservatively, nasogastric bowel decompression may be appropriate in some cases, the decision on whether surgical intervention or conservative treatment is used is critical in the management of patients with PI.

The severity of the underlying disease, intestinal ischemic changes seen on CT, and an Acute Physiology and Chronic Health Evaluation II (APACHE II) score above 18 points were reported to be factors that influence the prognosis of PI patients [8]. In the situation of a suspected life-threatening condition such as bowel ischemia, surgical intervention is mandatory. With regard to this, hypoperfusion conditions such as sepsis, use of inotropic agents, congestive heart failure, and other causes of hypotension are highly suspicious for ischemic bowel, and a low threshold for surgery should be observed [9].

Our case is interesting as the patient's PI was already diagnosed 7 years prior. He was asymptomatic, but after mechanical ventilation, secondary PI with small bowel volvulus occurred. Small bowel volvulus, is not a direct result of mechanical ventilation. It is suggested that edematous small bowel segment with large air-bubble stacked in subhepatic space, which caus- 
ed the torsion of mesenteric axis and subsequent small bowel obstruction.

What it not yet clear is the impact of mechanical ventilation on development of PI. As observed in this case, if there is a lung disease such as lung emphysema, alveoli may rupture due to coughing or mechanical pressure such as mechanical ventilation; the air from disrupted alveoli forms microbubbles and move to the mediastinum, gather there, and subsequently move together along the large vessels through the bloodstream, traveling to the retroperitoneum along the visceral vessels and penetrates the intestinal submucosa along the space around the vessel [3]. It is possible to hypothesize that these conditions are more likely to occur in patients who having emphysema treated with mechanical ventilation $[10,11]$.

As seen in our case, it is common for imaging studies, such as plain radiographs or CT scans, to show features suggestive of intraperitoneal free gas (Figure 1). This is thought to be a result of the perforation of individual gasfilled cysts, which may be asymptomatic. This case emphasizes the point that, although preoperative imaging is important in PI, clinical judgment is ultimately crucial in tackling this rare condition. It is important to identify that PI is a clinical sign and is in itself not a diagnosis. Because it represents such a wide variety of diseases, rapid evaluation of PI may be difficult. Although PI can be treated conservatively in some cases, surgical intervention should be performed in patients with PI presenting with signs of bowel obstruction or mesenteric torsion, after careful evaluation of the patients' current clinical state, comorbid conditions, physical examination, laboratory data, radiographic details, and diagnostic findings, such as in this case.

\section{CONFLICT OF INTEREST}

No potential conflict of interest relevant to this article was reported.

\section{ACKNOWLEDGMENTS}

The present study was supported by grants from Chosun University Hospital 2015.

\section{ORCID}

Dong Joon Kim http://orcid.org/0000-0002-3072-4734
Yong Joon Choi http://orcid.org/0000-0002-2899-8429

Young Sun Yoo http://orcid.org/0000-0002-0451-9788

\section{AUTHOR CONTRIBUTIONS}

Conceptualization: YSY. Data curation: YSY, DJK. Formal analysis: DJK, YJC. Funding acquisition: YSY. Visualization: YSY. Writing - original draft: DJK. Writing - review \& editing: YJC, YSY.

\section{REFERENCES}

1. Morris MS, Gee AC, Cho SD, Limbaugh K, Underwood S, Ham $\mathrm{B}$, et al. Management and outcome of pneumatosis intestinalis. Am J Surg 2008;195:679-82.

2. Koss LG. Abdominal gas cysts (pneumatosis cystoides intetsinorum hominis): an analysis with a report of a case and a critical review of the literature. AMA Arch Pathol 1952;53:523-49.

3. St Peter SD, Abbas MA, Kelly KA. The spectrum of pneumatosis intestinalis. Arch Surg 2003;138:68-75.

4. Heng Y, Schuffler MD, Haggitt RC, Rohrmann CA. Pneumatosis intestinalis: a review. Am J Gastroenterol 1995;90:174758.

5. Braumann C, Menenakos C, Jacobi CA. Pneumatosis intestinalis: a pitfall for surgeons? Scand J Surg 2005;94:47-50.

6. Jamart J. Pneumatosis cystoides intestinalis: a statistical study of 919 cases. Acta Hepatogastroenterol (Stuttg) 1979;26:41922

7. Greenstein AJ, Nguyen SQ, Berlin A, Corona J, Lee J, Wong E, et al. Pneumatosis intestinalis in adults: management, surgical indications, and risk factors for mortality. J Gastrointest Surg 2007;11:1268-74.

8. Kim HL, Lee HK, Park SJ, Yi BH, Ko BM, Hong HS, et al. Pneumatosis intestinalis: CT findings and clinical features. J Korean Radiol Soc 2008;58:149-54.

9. Donovan S, Cernigliaro J, Dawson N. Pneumatosis intestinalis: a case report and approach to management. Case Rep Med 2011;2011:571387.

10. Brant WE. Fundamentals of diagnostic radiology. 4th ed. Philadelphia: Wolters Kluwer Health; 2012.

11. Webb WR. Fundamentals of body CT. 2nd ed. Philadelphia: Elsevier Health Sciences; 2014. 